\title{
Modèle d'évolution des peuplements en futaie jardinée
}

\author{
M Bruciamacchie *, C Groualle, P Minot \\ ENITEF, domaine des Barres 45290 Nogent-sur-Vernisson, France
}

(Reçu le 3 septembre 1990; accepté le 18 décembre 1990)

\begin{abstract}
Résumé - Les futaies jardinées sont souvent étudiées par référence à des normes que l'on essaie de conserver après chaque coupe. C'est pourquoi les modèles d'évolution existants dans la littérature sont tous accompagnés d'une hypothèse implicite : conservation de l'accroissement en surface terrière, ou, dans le cas où l'on utilise des chaînes de Markov, que la matrice de transition reste constante.

S'appuyant sur une typologie des peuplements existante dans la région naturelle "Haut-Jura", le modèle proposé prend mieux en compte l'évolution constatée entre types de peuplement. Inspiré de la célèbre méthode du contrôle, il permet de réaliser des mises à jour d'inventaires. Il permet également d'entamer une réflexion sur la productivité des types de peuplement rencontrés et par conséquent de réaliser des simulations économiques. II est ainsi apparu que le type A évoluait rapidement vers le type $E$, et qu'il ne conservait sa stabilité qu'au détriment d'un prélèvement important dans les bois moyens.
\end{abstract}

futaie Jardinée / Haut-Jura / modèle d'évolution / méthode du contrôle / type de peuplement

Summary - Evolution model in populations of unevenly-aged stands. The unevenly-aged or selection stand is often studied in comparison with some norms that one tries to maintain after each felling, which explains why the existing evolution models in the literature imply conservation of the increasing basal area, or in cases when Markovian models are used, that the transition matrix remains constant. Based upon a stand typology existing in the natural region of the Haut Jura, the proposed model takes into account the evolution noted between stand types. Inspired by the Control Method, it enables inventories to be updated and also allows consideration of the productivity of the stand types encountered with resulting production of economic simulations. It therefore became apparent that type $A$ was in the process of rapidly evolving toward type $E$ and that it only conserved its stability to the detriment of the tree sampling in the half-mature stands.

selection forest / Haut-Jura / stand model / method of control / stand type

\footnotetext{
* Correspondance et tirés à part
} 


\section{INTRODUCTION}

II est particulièrement important dans des structures irrégulières (taillis-sous-futaie, futaie jardinée), de suivre la façon dont les arbres franchissent les différentes catégories de grosseur (évolution de la croissance en diamètre ou en circonférence). Le suivi de l'accroissement, telle fut l'idée directrice de Gurnaud (1878), idée traduite par la méthode du contrôle bien connue des forestiers Francs-Comtois, reprise par Biolley en Suisse en 1920 et qui a donné naissance aux USA à la méthode appelée "Continuous Forest Inventory" (Husch, 1963).

Cette méthode repose sur le calcul du pourcentage d'arbres qui franchissent au cours du temps les différentes catégories de diamètre, ce qui revient à établir une matrice de transition (probabilité de transfert d'une catégorie à une autre, ou de maintien dans la même catégorie). Elle est habituellement utilisée pour dresser un constat sur une période passée, et, dans le cas où elle sert à prédire l'histogramme futur d'un peuplement (Buongiorno, 1987), elle est souvent accompagnée d'une hypothèse implicite, qui est soit que la matrice de transition reste identique à elle-même si on raisonne par unité de temps constant, ou bien qu'il y a conservation de l'accroissement en surface terrière (Mertens et Gennart, 1985). Ces hypothèses découlent du fait que le jardinage est trop souvent présenté par référence à des normes sylvicoles que l'on essaie de conserver après chaque coupe.

De même, la plupart des calculs économiques effectués jusqu'alors sur les futaies jardinées (Remoussenard, 1984), reposent sur le principe que suite à une intervention humaine, chaque peuplement conserve son type. Cette hypothèse n'est que très rarement vérifiée. En effet, à cause d'un prélèvement généralement trop faible, ces peuplements évoluent d'un type à l'autre. II faut d'ailleurs conserver cette particularité des structures irrégulières, qui offrent à tout moment la possibilité de capitaliser ou de décapitaliser selon les besoins du propriétaire.

II nous est donc apparu nécessaire de proposer un modèle permettant de simuler l'évolution de l'histogramme d'un peuplement en prenant mieux en compte l'état initial du peuplement. Ceci sera réalisé en utilisant une méthode dérivée de la célèbre méthode du contrôle.

\section{MATÉRIEL ET MÉTHODES}

A titre d'exemple, nous allons développer le cas des futaies jardinées que l'on trouve dans la région naturelle "Haut-Jura". Herbert et Rebeirot $(1981,1985,1986)$ ont montré que ces peuplements pouvaient être décomposés en 7 types, évoluant les uns vers les autres. Leur étude, outre son intérêt en tant que base de réflexion sur la réalisation d'une typologie des peuplements, a permis de modifier cette perception du jardinage réduite trop souvent à la fameuse exponentielle que l'on trouve dans la plupart des manuels de sylviculture.

Le tableau I fournit les caractéristiques moyennes des types. II montre que ces derniers présentent des accroissements différents et auront donc des rentabilités spécifiques.

La figure 1 permet de visualiser les évolutions naturelles entre types. La sylviculture peut bien entendu modifier ces évolutions et permet par exemple d'aboutir à un type B.

\section{La méthode du contrôle}

Cette méthode est essentiellement utilisée par les gestionnaires pour suivre l'accroissement courant en volume sur une période, ainsi que le passage à la futaie en nombre. Elle est exposée 
Tableau I. Caractéristiques dendrométriques des différents types d'après Herbert et Rebeirot, 1981.

\begin{tabular}{|c|c|c|c|}
\hline $\begin{array}{c}\text { Nbre } \\
\text { tiges/ha }\end{array}$ & $\begin{array}{c}\text { Surface terrière } \\
m^{2} \cdot h a^{-1}\end{array}$ & $\begin{array}{c}\text { Volume/ha } \\
m^{3} \cdot h a^{-1}\end{array}$ & $\begin{array}{l}\text { Acct courant } \\
m^{3} \cdot h^{-1} \cdot a n^{-1}\end{array}$ \\
\hline
\end{tabular}

\begin{tabular}{lrcrrrrrr}
\hline A & $200-270$ & $16-20$ & $150-200$ & $45-50$ & $40-50$ & $5-10$ & 6,2 & 4,0 \\
B & $170-230$ & $18-27$ & $190-330$ & $30-45$ & $30-40$ & $20-35$ & 4,8 & 1,8 \\
C & $195-225$ & $12-16$ & $100-150$ & $45-60$ & $35-50$ & $0-10$ & 4,0 & 5,0 \\
D & $290-310$ & $18,5-22,5$ & $160-230$ & $45-55$ & $40-50$ & $2-8$ & & \\
E & $260-300$ & $22,5-26,5$ & $220-270$ & $30-35$ & $50-55$ & $10-20$ & 4,5 & \\
F & $180-260$ & $23-35$ & $250-400$ & $10-25$ & $35-45$ & $35-50$ & 4,4 & 0,9 \\
G & $60-120$ & $4,5-9,5$ & $40-100$ & & & & 1,8 & 1,6 \\
\hline
\end{tabular}

de manière très détaillée dans le célèbre ouvrage "Sapinières" de Schaeffer et al (1930). Nous allons simplement commenter quelques points essentiels.

Cette méthode se décompose en 2 phases.

\section{Établissement du tableau des promotions entre catégories de diamètre}

Connaissant les 2 inventaires d'une même parcelle à des instants $t_{1}$ et $t_{2}\left(t_{2}>t_{1}\right)$ ainsi que les coupes éventuelles, il est possible d'estimer le nombre d'arbres qui n'ont pas changé de catégorie durant la période (les stationnaires-S), ceux qui ont franchi une catégorie (les promusP), ceux qui ont franchi 2 catégories (les doubles promus-DP). II est possible d'imaginer, selon le même principe, l'existence de triples promus, etc. Les calculs assez simples développés ci-après sont adaptés aux inventaires par catégories de diamètre. Il est très facile de les transposer aux cas des inventaires par circonférence.

\section{Remarques}

- le tableau II se lit horizontalement, la diagonale correspondant aux stationnaires, puis on trouve successivement vers la droite, les promus, les doubles promus et ainsi de suite;

- il est possible de proposer d'autres tableaux des promotions. Si par exemple l'on augmente

Tableau II. Exemple de tableau des promotions - d'après Groualle et Minot, 1989.

$\begin{array}{llllllllllllll}N\left(t_{2}\right) & 41 & 41 & 35 & 32 & 35 & 32 & 13 & 4 & 1 & N\left(t_{1}\right) & \text { Diametre } & \begin{array}{l}\text { Station- Promus Double- } \\ \text { naires }\end{array} & \\ \text { Diamètre } & 20 & 25 & 30 & 35 & 40 & 45 & 50 & 55 & 60 & & & & \end{array}$

33

822

1932

331

135

0325

08

$\begin{array}{llll}0 & 3 & 1 & 4\end{array}$

0

$\begin{array}{rrrrr}30 & 20 & 8 & 22 & \\ 51 & 25 & 19 & 32 & \\ 34 & 30 & 3 & 31 & \\ 36 & 35 & 1 & 35 & \\ 37 & 40 & 0 & 32 & 5 \\ 9 & 45 & 0 & 8 & 1 \\ 4 & 50 & 0 & 3 & 1 \\ & 55 & & & \end{array}$




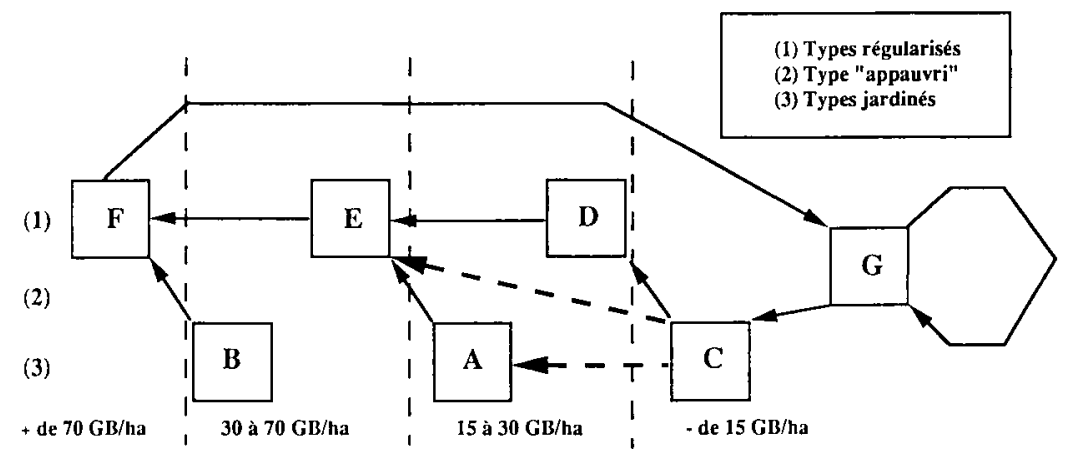

Fig 1. Evolution des types de peuplement du Haut-Jura. Extrait de Herbert et Rebeirot (1985). Remarque : Les flèches en pointillés correspondent aux évolutions proposées par Groualle et Minot (1989).

le nombre de doubles promus d'une catégorie, cela conduit à diminuer le nombre de promus de la (ou des) catégorie supérieure. La méthode du contrôle contient donc l'hypothèse qu'il est plus probable qu'un arbre franchisse une seule catégorie de diamètre plutôt que deux;

- s'il y a eu coupe entre les 2 inventaires à l'instant $t^{\prime}\left(t_{1}<t^{\prime}<t_{2}\right)$, il est nécessaire de prendre en compte les arbres qui ont été prélevés. Cela se réalise habituellement en soustrayant à l'inventaire initial et en ajoutant à l'inventaire final le nombre d'arbres de la coupe, au prorata du temps écoulé entre la coupe et les inventaires. On obtient ainsi des inventaires ajustés;

- ce tableau des promotions dépend de la durée entre les 2 inventaires, de la vitesse de croissance des arbres et du peuplement initial.

\section{Acroissement par catégorie de dia- mètre}

Possédant la répartition des arbres en (S), (P), (DP), (TP), il est possible d'en déduire l'accroissement sur le diamètre, en surface terrière, en volume, ainsi que les temps de passage par catégorie de diamètres. Pour ce faire, on suppose que les stationnaires n'ont eu aucun accroissement, que les promus ont tous eu une croissance égale à $\Delta$, les doubles promus à $2 \Delta$, etc. ( $\Delta$ étant l'amplitude des classes de diamètre). Ces hypothèses valables en moyenne, ne se justifient que si le nombre d'arbres par catégorie de diamètre est suffisant $(>20)$.

Cette hypothèse étant faite, il existe 2 manières différentes de calculer l'accroissement. Pour la suite du texte, les notations suivantes seront utilisées.

$N d$ : Nombre d'arbres de la catégorie $d ; N d_{1}$ : Nombre d'arbres de la catégorie $d$ à l'instant $t_{1}$; $\mathrm{Nd}_{2}$ : Nombre d'arbres de la catégorie $d$ à l'instant $t_{2} ; S d$ : Nombre d'arbres stationnaires de la catégorie $d ; P d$ : Nombre d'arbres de la catégorie $d$, promus à la catégorie $d+1 ; D P d$ : Nombre d'arbres de la catégorie $d$, promus à la catégorie $d+2$; $I D d$ : Accroissement moyen sur le diamètre de la catégorie $d ; T d$ : Temps de passage de la catégorie $d ; R$ : Période séparant 2 inventaires (Rotation).

$1^{r \theta}$ méthode

On suit l'évolution des arbres appartenant initialement à une même catégorie. Dans ce cas, l'accroissement annuel sur le diamètre devient par exemple:

$$
I D_{d}=\frac{\Delta}{R \cdot N_{d}}\left(P_{d}+2 D P_{d}+3 T P_{d}+\ldots\right)
$$

Ce procédé de calcul a été exposé par Vaulot (1914), ou plus récemment par Mertens et Gennart (1985).

\section{$2^{\circ}$ méthode}

On considère une catégorie de diamètre, et on observe les arbres qui transitent par cette caté- 
gorie. II est nécessaire de calculer un effectif moyen sur la période par catégorie $\left(\left(\mathrm{Nd}_{1}+\right.\right.$ $\mathrm{Nd}_{2}$ )/2), ainsi que le nombre d'arbres ayant transité par la catégorie, moyenne des arbres étant partis de la catégorie et ceux étant arrivés dans la catégorie.

$$
1 D_{d}=\frac{\Delta}{R} \frac{\left(P_{d}+2 D P_{d}+\ldots+P_{d-1}+2 D P_{d-2}+\ldots\right)}{\left(N_{d 1}+N_{d 2}\right)}
$$

Cette méthode est exposée dans Schaeffer et al (1930).

Ces 2 méthodes testées sur une cinquantaine de parcelles conduisent à des résultats proches. Si le nombre d'arbres par catégorie est faible, la deuxième méthode est à conseiller car l'accroissement est alors calculé sur un nombre d'arbres supérieur. À l'inverse pour la première catégorie de diamètre, il convient de conseiller la première méthode.

De la même façon que pour l'accroissement sur le diamètre, il est possible de fournir les formules permettant de calculer l'accroissement en surface terrière (Mertens et Gennart, 1985).

Le temps de passage par catégorie de diamètre est également une variable très utilisée en futaie jardinée. II en existe plusieurs définitions :

- un nombre d'années que met l'arbre moyen de chaque catégorie à franchir les limites de cette catégorie (Schaeffer et al, 1930);

- nombre d'années que met un arbre pour franchir une catégorie de grosseur (Mertens et Gennart, 1985).

Nous retiendrons cette dernière définition. Cela se traduit par exemple dans le cas de la $1^{\text {re }}$ méthode par la formule suivante :

$$
T_{d}=\frac{\Delta}{I D_{d}}=\frac{R \cdot N_{d}}{P_{d}+2 D P_{d}+\ldots}
$$

\section{RÉSULTATS}

\section{Temps de transition entre les types}

Les résultats précédents vont être utilisés pour calculer les durées d'évolution entre types. Cette fois l'inconnue de la formule (i) n'est plus $T d$, mais $R$, qui est le temps nécessaire à la transition entre deux types. Les nombres de stationnaires, de promus, de doubles promus, sont calculés en prenant comme premier inventaire la norme du type de départ, et comme deuxième inventaire la norme du type d'arrivée. Il est nécessaire également d'avoir des informations sur l'accroissement moyen des arbres, par l'intermédiaire des temps de passage ou des accroissements sur le diamètre.

Dans le cas des temps de passage, nous retiendrons la moyenne des temps de passage des types de départ et d'arrivée.

La formule utilisée est donc :

$$
R_{d}=\frac{\left(T_{d i}+T_{d f}\right)}{2} \frac{\left(P_{d}+2 D P_{d}+\ldots\right)}{N_{d}}
$$

Le tableau III traite le cas du passage du type $A$ au type $E$ (les temps de passage utilisés sont ceux calculés par Herbert et Rebeirot, 1985). La rotation nécessaire au passage entre 2 types est en fait la moyenne pondérée par les effectifs de chaque classe de diamètre, des $R_{d}$ calculés pour chaque classe de diamètre. Pour le passage du type $A$ au type $E$ cette moyenne est égale à 9 ans.

La figure 2 fournit les temps de passage entre toutes les évolutions naturelles prévues par Herbert et Rebeirot (1985) mais aussi pour les cas rencontrés dans les inventaires à notre disposition.

Remarque : ces temps de passage ne sont valables que si on laisse évoluer le peuplement sans intervention. Ils correspondent à une durée minimale. II est à noter que les peuplements à faible matériel sur pied tels que le $C$ et le $A$, ont des durées d'évolution très faibles. 
Tableau III. Rotation nécessaire pour passer du type A au type E d'après Groualle et Minot, 1989.

\begin{tabular}{|c|c|c|c|c|c|c|c|}
\hline Diamètre & Type A & Type E & Stationnaire & Promus & Passage à la futaie & $T$ & R \\
\hline 20 & 68 & 51 & 32 & 36 & 19 & 19 & 10 \\
\hline 25 & 55 & 50 & 14 & 41 & & 16 & 12 \\
\hline 30 & 50 & 62 & 21 & 29 & & 14 & 8 \\
\hline 35 & 40 & 50 & 21 & 19 & & 13 & 6 \\
\hline 40 & 20 & 32 & 13 & 7 & & 14 & 5 \\
\hline 45 & 11 & 12 & 5 & 6 & & 11 & 6 \\
\hline 50 & 3 & 7 & 1 & 2 & & 12 & 8 \\
\hline 55 & 1 & 2 & & $\overline{1}$ & & 16 & 16 \\
\hline 60 & & 1 & & & & & \\
\hline Somme & 248 & 267 & & & & \multicolumn{2}{|c|}{ moyenne : 9} \\
\hline
\end{tabular}

Les chiffres mentionnés en gras sont les termes calculés.

\section{Mise a jour d'inventaires}

Les méthodes exposées ci-après ont pour but de prévoir l'évolution des peuplements sur des périodes relativement courtes. De nombreux gestionnaires se sont intéressés au problème de la mise à jour d'inventaires, et il existe donc une multitude de méthodes. Nous n'en proposerons que 3 , les 2 premières extraites de Mertens et Gennart (1985), la troisième de Groualle et Minot (1989).

\section{Première méthode}

Hypothèse de conservation

des conditions de croissance

Le tableau des promotions, obtenu grâce à 2 inventaires antérieurs, sera appliqué tel quel sur la nouvelle période. Cela suppose que la durée de mise à jour est égale à la rotation antérieure, et qu'une coupe éventuelle a ramené le peuplement dans son état initial.

\section{Deuxième méthode Hypothèse de conservation de l'accroissement en suface terrière par catégorie de diamètre}

Cette hypothèse s'applique dans le cas où it y a modification des conditions locales de concurrence (changement de type de peuplement), ou différence entre la durée d'actualisation $\left(t_{4}-t_{3}\right)$ et la rotation entre les 2 inventaires antérieurs $\left(t_{2}-t_{1}\right)$. II s'agit dans ce cas de recalculer un nouveau tableau des promotions, à l'aide de la croissance en surface terrière et du passage à

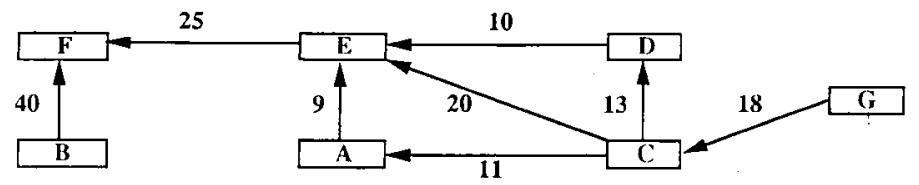

Fig 2. Temps de transition entre types (années). 
la futaie extraits du premier intervalle (entre $t_{1}$ et $t_{2}$ ).

\section{Troisième méthode Modélisation de l'accroissement en futale jardinée.}

Les deux méthodes précédentes sont bien adaptées aux parcelles en équilibre, car elles prennent en compte l'accroissement local lié à la station. Par contre, elles nécessitent la connaissance de 2 inventaires antérieurs. Ces conditions n'étant généralement pas remplies, il nous a semblé intéressant de rechercher un modèle d'évolution qui puisse prendre en compte des durées d'actualisation variables. $\mathrm{Ce}$ modèle a été obtenu en utilisant 47 inventaires à notre disposition.

II a été obtenu par régression pas à pas. Il se réduit à un système de 5 équations (tableau IV). Les 2 premières fournissent l'accroissement du peuplement (NPF, IG) en fonction des variables décrivant le peuplement initial (N/ha, G/ha, PB, BM, $\mathrm{GB})$. Les 3 dernières permettent de calculer les temps de passage des différentes catégories de diamètre.
Ce système d'équations permet de calculer un tableau de promotions à partir d'un inventaire initial et d'une durée souhaitée de mise à jour. Si on note $R$ cette période d'actualisation, alors,

$$
\begin{aligned}
& D P_{d}=0 \\
& \text { si } \frac{R}{T_{d}} \leq 1 \text { alors } P_{d}=\frac{N_{d} R}{T_{d}} \\
& S_{d}=N_{d}-P_{d} \\
& \mathrm{R} \quad \mathrm{T}_{\mathrm{d}} \\
& \text { si } 1 \leq-\leq 2 \text { alors } P_{d}=N_{d}-D P_{d} \\
& T_{d} \quad S_{d}=0
\end{aligned}
$$

Les calculs seront faits par itérations de 5 ans, ce qui évitera d'avoir des triples promus.

Ce modèle a été testé sur 39 inventaires, en comparant la surface terrière et le nombre de tiges fournis par le modèle, aux résultats réels. La figure 3 montre que dans la grande majorité des cas les erreurs sont inférieurs à $10 \%$, et qu'il n'y a

Tableau IV.

$\begin{array}{lll}N P F=-23,75+0,312 P B+0,179 B M+0,3597 \mathrm{~GB}+0,0409 \mathrm{~N} / \mathrm{ha}-0,463 \mathrm{G} / \mathrm{ha} & 0,742 & 1,51 \\ I G=-0,3804+0,00718 P B+0,0214 \mathrm{~GB}+0,00593 \mathrm{~N} / \mathrm{ha}-0,05691 \mathrm{G} / \mathrm{ha} & 0,795 & 0,135 \\ T B M=26,55+0,0471 \mathrm{~N} / \mathrm{ha} \pm 49,77 \mathrm{IG}+2,47 \mathrm{NPF}-62,07 / G / \mathrm{ha} & 0,843 & 3,72 \\ T P B=22,542+0,0464 \mathrm{~N} / \mathrm{ha}-2,199 \mathrm{NPF}-1,810 \mathrm{TBM}+0,0794 \mathrm{TBM} & 0,885 & 7,5 \\ T G B=0,37+0,974 \mathrm{TBM} & 0,687 & 6,68\end{array}$

où $N P F=$ passage à la futaie en nombre de tiges par hectare et par an; $/ G$ = accroissement en surface terrière (en $\mathrm{m}^{2} / \mathrm{ha}^{-1 / a n^{-1}}$ ); TPB : temps de passage des petits bois (20-25); TBM = temps de passage des bois moyens (3040); TGB = temps de passage des gros bois (45 et + ); Nha :=densité initiale du peuplement; $G / h a$ = surface terrière initiale du peuplement; $P B=$ pourcentage initial de petits bois; $B M=$ pourcentage initial de bois moyens; $G B=$ pourcentage initial de gros bois; $\sigma=$ ecart type résiduel. 


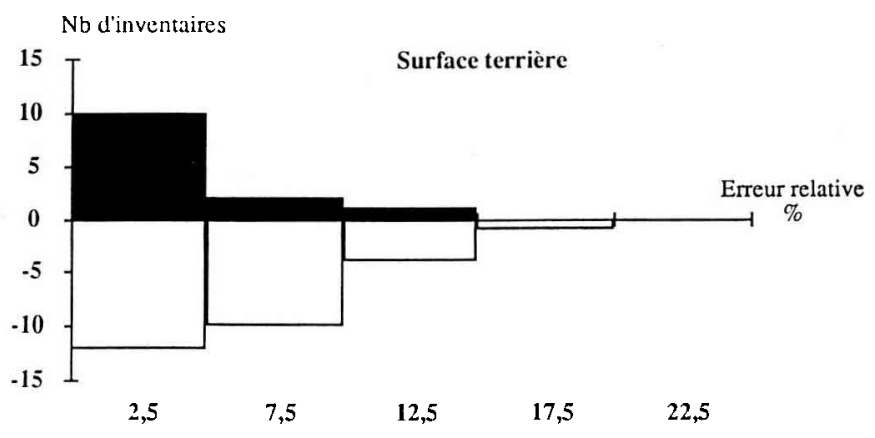

$\mathrm{Nb}$ d'inventaircs

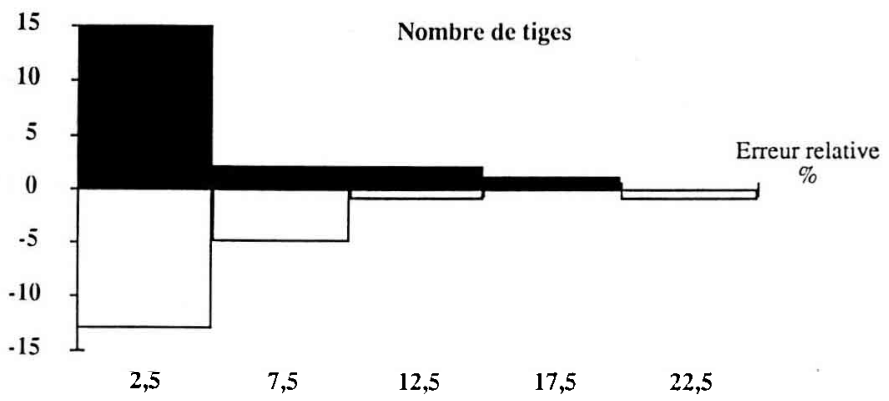

Fig 3. Validation du modèle proposé.

pas de sous ou de surestimation systématique.

Ce modèle peut également être testé en introduisant comme histogramme initial, l'un des types de peuplement. Le ta- bleau $V$ fournit les accroissements sur le volume et le passage à la futaie en nombres estimés par le modèle. Ces estimations peuvent être comparées pour les types $A, E$ et $B$ aux valeurs fournies par le

Table V. Accroissement sur le volume et passage à la futaie estimé par le modèle.

\begin{tabular}{lccccc}
\hline & \multicolumn{5}{c}{ Accroissement sur le volume $\left(\mathrm{m}^{3} . \mathrm{ha}^{-1} . \mathrm{an}^{-1}\right)$} \\
& Vol Total & Vol GB & Vol BM & Vol PB & NPF \\
\hline Type A & 5,9 & 3,2 & 2,6 & 0,2 & 3,8 \\
Type A40 & 4,9 & 2,9 & 1,9 & 0,1 & 2,8 \\
Type E & 3,7 & 2,8 & 1 & $-0,1$ & 1,6 \\
Bellefontaine & 6,5 & 3,1 & 3,4 & $-0,1$ & 4,1 \\
Type B & $\mathbf{3 , 5}$ & 2,9 & 0,4 & 0,1 & 1,7 \\
Type B(1) & 3,9 & 3,2 & 0,5 & 0,2 & 1,7 \\
\hline
\end{tabular}

(1) Ce peuplement a été cubé en utilisant un Alan 11; tous les autres peuplements sont cubés avec un Algan 9. 
Tableau VI. Recherche du type idéal.

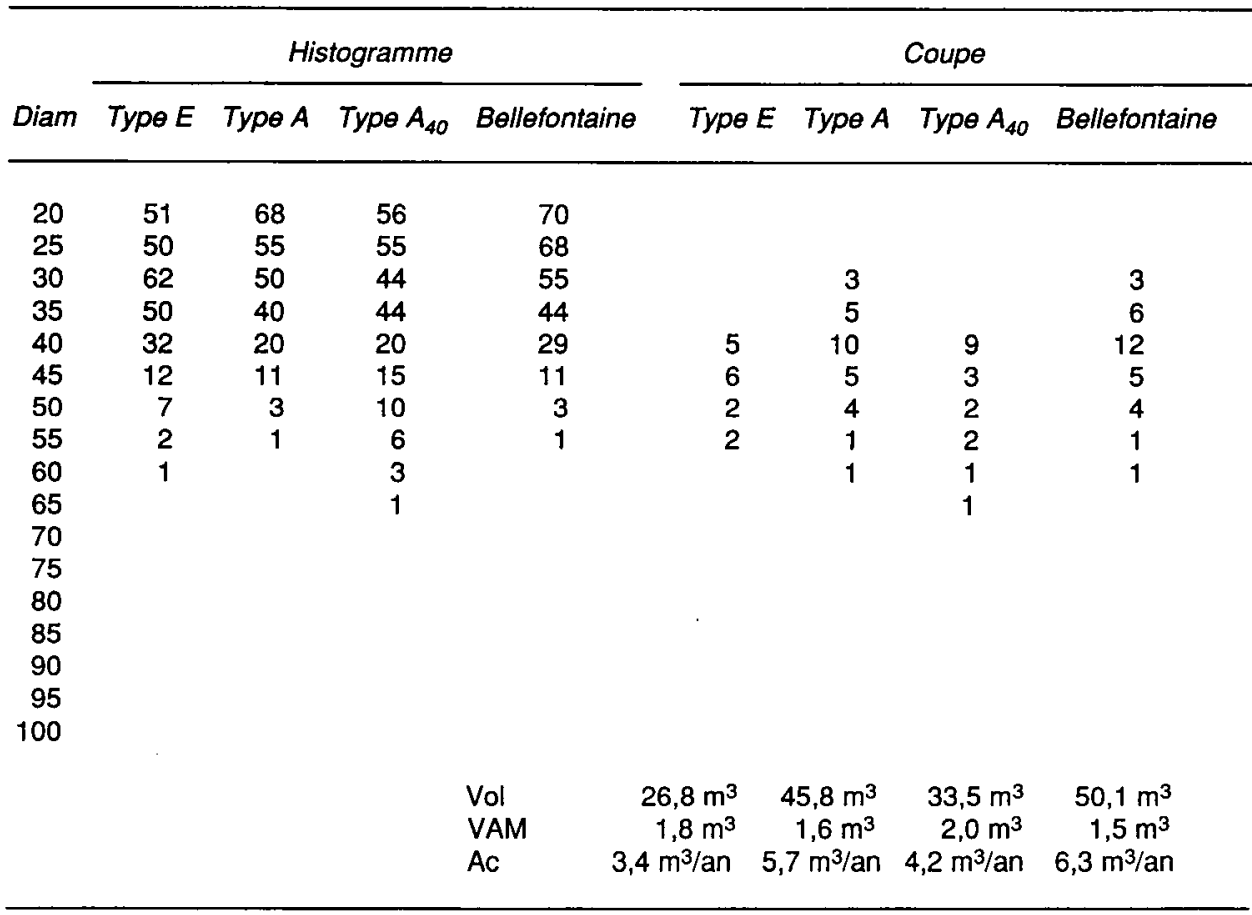

tableau I. On constate une assez bonne estimation du passage à la futaie. Les valeurs de l'accroissement sur le volume, systématiquement inférieures, soulèvent le problème du choix du tarif qui n'est sans doute pas identique à ceux utilisés par Herbert et Rebeirot (1981).

\section{DISCUSSION}

Le modèle proposé peut être utilisé pour comparer les types A (considéré comme idéal par Herbert et Rebeirot), E (le plus représenté en surface), $A_{40}$ (proposé par Groualle et Minot), ainsi que la parcelle 26 du canton de Bellefontaine qui est un exemple de peuplement de type A possé- dant une production assez élevée. Le tableau VI fournit les histogrammes des 4 types de peuplement comparés, ainsi que le nombre de tiges à prélever en coupe tous les 8 ans pour les ramener dans leurs états initiaux.

On constate que le type A présente une production élevée, mais que le volume de l'arbre moyen prélevé en coupe est faible. Or, on sait que le coût d'une exploitation est directement lié au volume de l'arbre moyen. Le type $A$ ne se maintient donc qu'au prix d'un prélèvement important dans les bois moyens. Le sylviculteur ayant à juste titre des scrupules à prélever dans ces catégories de diamètre, c'est sans doute, avec le fait que le type $A$ évolue assez rapidement ( 9 ans) vers le type $E$, 
une des raisons qui explique la faible répartition en surface du type $A$.

Le type $A_{40}$ semble être un bon compromis entre un volume de l'arbre moyen élevé $\left(2 \mathrm{~m}^{3}\right)$ et une production satisfaisante.

\section{CONCLUSION}

La méthode du contrôle proposée par Gurnaud, il y a plus d'un siècle, décompose les arbres en stationnaires, promus, etc. Cette méthode déjà ancienne nous a permis de calculer les temps de passage entre les types de peuplements du HautJura. II est ainsi apparu, que le type A évoluait rapidement vers le type $E$ et qu'il ne conservait sa stabilité qu'en prélevant un grand nombre de bois moyens. C'est ce qui explique sans doute sa faible importance en surface.

Elle a également inspiré la création d'un modèle permettant d'actualiser des inventaires, de prédire l'évolution d'un peuplement suite à une coupe, et par conséquent de réaliser des simulations économiques.

Le modèle proposé a pour seule ambition de contribuer à l'étude des peuplements irréguliers. II montre en particulier qu'il est préférable de raisonner non pas en production totale, mais plutôt en production par catégories de produits. II a également permis d'entamer une réflexion sur ce que doit être un peuplement idéal : il doit avoir un passage à la futaie suffisant, une production élevée et concentrée dans les gros bois, il doit être stable (une coupe doit le ramener dans son état ini- tial), et le volume de l'arbre moyen prélevé en coupe doit être le plus élevé possible.

\section{RÉFÉRENCES}

Biolley H (1920) L'aménagement des forêts par la méthode expérimentale et spécialement la méthode du contrôle. Attinger frères, Neuchâtel et Paris, $85 \mathrm{p}$

Buongiorno J, Kaya I (1987) Economic Harvestiong of Uneven-Aged Northern Hardwood Stands Under Risk: a Markovian Decision Model. For Sci 33, 4, 889-907

Groualle C, Minot P (1989) Croissance du sapin et de l'épicéa dans les futaies jardinées du Haut-Jura. Mémoire ENITEF, $69 p$

Gurnaud A (1878) Cahier d'aménagement pour l'application de la méthode par contenance exposée sur la forêt des Eperons, J Temblay, Paris, $160 p$

Herbert I, Rebeirot F (1981) Les futaies jardinées privées des hautes chaînes du Jura. Mémoire ENITEF, $121 \mathrm{p}$

Herbert I, Rebeirot F (1985) Les futaies jardinées du Haut-Jura, $1^{\text {re }}$ partie. Rev For Fr 6 , 465-481

Herbert I, Rebeirot F (1986) Les futaies jardinées du Haut-Jura, $2^{\theta}$ partie. Rev For Fr 6 , 564-572

Husch B (1963) Forest Mensuration and Statistics, The Ronald Press Co, New York, $474 \mathrm{p}$

Mertens P, Gennart M (1985) Gestion de la futaie jardinée. Un modèle simple de calcul des accroissements et de simulation de la production. Bull Soc Roy For Belg 3, 105-123

Remoussenard P (1984) Étude pratique et économique des futaies jardinées des hautes chaînes du Jura. Mémoire ENITEF, $127 p$

Schaeffer A, Gazin A, D'Alverny A (1930) Sapinières. PUF, Paris, $100 p$

Vaulot G (1914) Détermination des accroissements en diamètre des arbres. Rev For Fr, 145-154 